Aims. Hyperprolactinaemia is a problem secondary to antipsychotic use. Current management guidelines are heterogeneous and impractical. We aimed to assess coherence to common themes monitoring and intervention, reasons for failure, and to design new guidance for both general use Barnet, Enfield and Haringey Mental Health Trust (BEHMHT) and beyond.

We hypothesised that performance would be poor and new guidance warranted.

Background. Hyperprolactinaemia is defined as blood prolactin of $>530 \mathrm{miu} / \mathrm{L}$ in females and $>424 \mathrm{miu} / \mathrm{L}$ in males, with $49.9 \%$ is due to medication. Several agents are deemed higher risk Symptom profiles and risk are idiosyncratic and there are adverse long-term outcomes. Treatment is based on symptom profile and severity and cause. Current guidance is trust specific or advised through The Maudlsey Prescribing Guidelines.

Comprehensive and practical guidance reflecting front-line limitations is lacking. There is no clear delineation of a risk stratified pathway.

Method. We wished to ascertain data on surveillance, aetiology and signpost opportunities for service improvement. We also designed 'risk strata' to guide intervention.

A random sample (n30) was selected from Enfield South Locality Team and data captured using local records. No ethical considerations were raised.

A number of audit standards (95\%) were developed based on previous guidance and agreed within the team and included frequency of monitoring, time to review and need for further referral.

New guidance was developed based on results, MDT agreement and consultation with medical specialities.

Result. Data (n 30) showed predominant male bias to sample $(66 \%)$ and average age of 48.87 yrs. Predominant diagnoses were Paranoid Schizophrenia (53.33\%) and Schizoaffective disorder $(33.33 \%$.) Only $7 / 30$ (23.33\%) had undergone testing within the last year.

Of those sampled, 2 (6.667\%) had a new diagnoses of Hyperprolactinaemia, one on routine monitoring, one incidentally on admission to hospital. Both were on high risk agents. Both were reviewed and treated within one month. No audit standards were met, but no further referrals were required.

Reasons for failure varied, but included loss to follow-up, no test requested or appointments missed.

Conclusion. Based on these data it was noted that monitoring was poor and reasons for failure varied. New Guidance was developed in response. The scope and validity of this guidance was agreed by MDT and awaits formal ratification.

Re-audit will occur in 2020, and if successful the guidance submitted to other Trusts and RCPSYCH for national use.

No financial interests to declare.

\section{Using SBAR in psychiatry: findings from two london hospitals}

Benjamin Janaway ${ }^{1 \star}$, Ruslan Zinchenko ${ }^{1}$, Lubna Anwar ${ }^{2}$, Clare Wadlow ${ }^{1}$, Edwin Soda ${ }^{1}$ and Karen Cove ${ }^{1}$

${ }^{1}$ Barnet, Enfield and Haringey Mental Health Trust and ${ }^{2}$ Enfield South Locality Team (Lucas House)

${ }^{\star}$ Corresponding author.

doi: 10.1192/bjo.2021.530

Aims. We aimed to evaluate the use of the Situation, Background, Assessment and Recommendation communication tool (SBAR) at two large psychiatric hospitals, in order to design new approaches to teach and reinforce its sustained use. In doing so we hope to improve communication, staff experience and outcomes for patients.

We hypothesised that use prior to intervention would be low and attitudes inconsistent between teams and objective data.

Background. SBAR is a communication tool developed to accurately refer information with improved outcomes within the NHS. Within psychiatry there is evidence of relatively poor care of medical problems leading to adverse outcomes in a group more susceptible to multiple physical illnesses. The reasons for this include a cultural ethos of learned helplessness in staff and lack of medical knowledge.

The use of SBAR is likely to overcome these issues.

Method. Surveys were presented to doctors and nurses staff at two Psychiatric Hospitals, Chase Farm and Edgeware. Inclusion in the survey was voluntary and anonymous. Questions elucidated topics ranging from awareness of SBAR through to its use and benefits.

Objective data were also collected, looking at handover gathered during the survey period. This was collected via phone from the duty physician over a five-day period, twice-daily. Qualitative data on handover content was collected at CFH.

Audit standards around knowledge, use and outcomes were set. Data were collected and analysed in house.

Result. The data (n23) showed that most nurses reported awareness $(86.96 \%)$ ease of use $(86.96 \%)$ actual use $(60.87 \%)$ efficacy in communication $(78.26 \%)$ value in understanding patients (78.26\%) and agreement with mandatory use $(78.26 \%$.)

Doctor reports (n14) showed that although $100 \%$ were aware of SBAR, no respondents thought nurse-led communication was adequate, or that SBAR was used. The majority thought that mandatory SBAR use would improve communication (92.86\%) and patient care (100\%)

Objective data (pooled) of referrals showed that on $6.52 \%$ used SBAR. Qualitative data showed that handover was often inaccurate, lacking in information and unsafe. Suggestions for teaching included written or video media, or taught classes.

All audit standards were failed.

Conclusion. SBAR is an effective tool for improving communication and patient outcomes, and is well perceived by the MDT. However, it is poorly used with psychiatry leading to adverse outcomes. Reported use is undermined by objective data. Its mandatory use is well supported and new teaching initiatives are thus being designed to remedy this and improve client experience.

\section{Quality improvement project: improving the confidence of junior doctors to manage emergencies; Drs abc in an acute psychiatric setting \\ Ahrane Jayakumar ${ }^{1 \star}$, Wren Erin-Jones ${ }^{2}$ and Simon Edwards ${ }^{3}$ \\ ${ }^{1}$ Gordon Hospital (CNWL); ${ }^{2}$ St Mary's Hospital, CNWL and \\ ${ }^{3}$ Medical Director and Consultant Physician, Diggory Division, CNWL \\ ${ }^{\star}$ Corresponding author.}

doi: 10.1192/bjo.2021.531

Aims. To improve the confidence and preparedness of junior doctors in managing medical or psychiatric emergencies when on call at an inpatient psychiatric unit.

Background. Facilities for emergency care differ between acute medical and psychiatric units. Protocols for managing acutely deteriorating patients and those requiring immediate resuscitation differ across these organisations.

Managing medical emergencies can be stressful for all involved. Junior doctors rotate between services where the level 
of support varies depending on specialty and setting. For doctors who have worked in a setting where the minimum emergency response includes a Resuscitation team, moving to an environment with less support available is a challenge.

In our unit, the protocol following an urgent call is for the on-call doctor (who has access to basic resuscitation equipment) to attend and assess the need for paramedics and transfer to local hospital. Stress can be worsened by change of environment, change of expectation and concern about best management in new settings.

Method. A cohort of junior doctors were recruited. Baseline assessment included rating their confidence level (scale 1- 10), listing common medical and psychiatric scenarios they had experienced and those they felt least confident managing. Over a period of 10 weeks, follow-up data was obtained. Interventions to improve confidence were assessed during this period, including a handbook and a teaching session on emergency medications. At the end of the project a wordcloud was created in response to the request to "choose 5 words to describe your feelings when called to an emergency". Identified themes have been fed back to relevant senior staff and will form the basis of future projects.

Result. The initial average confidence score improved from 4.9 to 9.2 and was sustained out to 14 weeks. According the word cloud the most commonly used words were "morale" and "education". Conclusion. Prior to the study, confidence levels amongst the Junior Doctors was low. Introduction of the handbook and teaching session led to an improvement which was sustained. Key themes identified using a word cloud were "morale" and "education".

For junior doctors moving from between services, different expectations and protocol for management of emergencies can influence confidence levels. Psychiatric units should be cognisant of these concerns and implement evidence-based intervention to support junior doctor confidence and improve quality of working experience.

Perception of junior doctors about local induction in Birmingham and Solihull Mental Health Foundation Trust - A quality improvement project

Saima Jehanzeb ${ }^{1 \star}$, Kozara Nader ${ }^{2}$ and Ruth Scally ${ }^{2}$

${ }^{1}$ Birmingham and Solihull Mental Health Foundation Trust and

${ }^{2}$ Birmingham and Solihull Mental Health NHS Foundation Trust

${ }^{\star}$ Corresponding author.

doi: 10.1192/bjo.2021.532

Aims. A quality improvement project was undertaken to understand the perception of trainees about the quality of the local induction delivered by Birmingham and Solihull Mental Health Foundation Trust (BSMHFT). The primary aim was to evaluate the current local induction programme, following concerns raised by previous trainees in National Training Survey (General Medical Council) and local inspection. Our secondary aim was to devise a revised induction programme based on the trainees' identified needs.

Method. Two anonymised questionnaire surveys were emailed to all Foundation Year Trainees, Core Psychiatry Trainees and General Practice Speciality Trainees working in BSMHFT, in December 2019 and March 2020, using trust survey monkey.

Result. The overall response to survey was 60 percent. 44.44 percent of the responses came from Core Psychiatry Trainees, with
27.78 percent responses each from Foundation Year Trainees and GP Speciality Trainees. Local induction was defined as induction specific to place of work (47.06\%), trust based induction (41.18\%) or all of the above options (11.76\%) by trainees. $83.33 \%$ of all trainees had received local induction, whereas $16.67 \%$ did not have any local induction at the start of their post. $11.12 \%$ trainees were very satisfied and $44.44 \%$ were satisfied with local induction. 72.22 percent of the trainees were informed about of the local induction, prior to starting the post.

$33.3 \%$ trainees had a paper version, $22.22 \%$ had an electronic version of local induction pack, whereas $44.44 \%$ had no induction pack. $55.55 \%$ of those trainees who had an induction pack, $43.75 \%$ found it very helpful and $56.25 \%$ did not find it helpful.88.89\% thought having a local induction would be helpful, whereas 11.11 percent did not feel it would help. $94.44 \%$ of the trainees completed a local orientation checklist with their consultants. Some of the trainees experienced difficulty in gaining access to electronic prescribing, electronic patient record system (RIO), and identity badges (ID) at the beginning of their post.

Conclusion. $11.12 \%$ trainees were very satisfied, $44.44 \%$ were satisfied, $22.22 \%$ were neither satisfied nor dissatisfied and $22 \%$ were dissatisfied, with local induction. $88.89 \%$ of the trainees thought having a local induction pack would be helpful. Based on the trainees identified needs we developed a template for local induction pack for each post. Clinical supervisors have agreed to take the lead in preparing the local induction pack specific to their post with trainees.We aim to repeat the survey after implementing the changes identified by trainees based on their training needs.

\section{Planning effective mental healthcare in prisons: findings from a national consultation on the care programme approach in prisons}

Jemini Jethwa* and Kate Townsend

The Royal College of Psychiatrists

${ }^{*}$ Corresponding author.

doi: $10.1192 /$ bjo.2021.533

Aims. The Care Programme Approach (CPA) can be an effective tool in coordinating the care and treatment needs of people with mental illness and learning disabilities. Within prisons settings, the CPA has been poorly implemented and the principles underpinning this approach have been lost. The aim of this research was to look at the key themes identified as part of a consultation process to develop quality guidance on planning effective mental healthcare in prisons in relation to the CPA.

Method. The consultation exercises included telephone interviews and hosting a national consultation event to represent the views of prisons nationally. It was conducted by the Quality Network for Prison Mental Health Services, a quality improvement initiative organised by the Royal College of Psychiatrists' Centre for Quality Improvement.

Result. The results derived from the consultation process indicates that $\mathrm{CPA}$ in prisons is inconsistently adopted and that there is lack of confidence in the process from prison mental health teams, particularly with how to engage community mental health teams.

Conclusion. This concludes that there is a substantial need for standardisation and consistency in the application of the CPA process within prisons, for the purposes of enhanced care delivery, greater continuity of care, and improved patient outcomes. 